\title{
The role of early determination of $\beta$-human chorionic gonadotropin levels in predicting the success of single-dose methotrexate treatment in ectopic pregnancy
}

\author{
Handan Çelik ${ }^{1}$, Miğraci Tosun ${ }^{1}$, Yüksel Işık1 , Bahattin Avcı² \\ 'Department of Obstetrics and Gynecology, University of Ondokuz Mayıs, School of Medicine, Kurupelit, Samsun, Turkey \\ ${ }^{2}$ Department of Biochemistry, University of Ondokuz Mayıs, School of Medicine, Kurupelit, Samsun, Turkey
}

\begin{abstract}
Objective: The aim of the study was to determine the importance of $\beta$-hCG level on day 4 following methotrexate (MTX) administration, and the difference between $\beta$-hCG levels assessed on day 0 and day 4 in predicting treatment success.

Material and methods: A total of 68 women with tubal pregnancy, treated with a single dose of MTX, were selected for this retrospective study.

Results: The success rate of single-dose MTX treatment in our clinic was 75\% (51/68). Among 51 patients in whom MTX treatment was successful, 25 (36.8\%) showed a decrease in $\beta$-hCG level of $>15 \%$ on days 0 and 4 , and 44 (64.7\%) showed a $\beta$-hCG level decrease of $>15 \%$ on days 4 and 7 . For subjects with $\beta$-hCG decrease of $>15 \%$ on days 4 and 7 , the standard error was $6.5 \%$, and the area under the ROC curve was $81.7 \%$, while the corresponding values for days 0 and 4 were $7.2 \%$ and $64 \%$, respectively.

Conclusions: A decrease of $>15 \%$ in $\beta$-hCG levels between days 0 and 4 does not seem to be a better predictor for success of single-dose MTX treatment for ectopic pregnancy than between days 4 and 7. A statistically significant difference was observed only in $\beta$-hCG levels on day 7 in both, successful and unsuccessful single-dose MTX groups.
\end{abstract}

Key words: ectopic pregnancy, methotrexate, single dose, human chorionic gonadotropin, treatment success

\section{INTRODUCTION}

The incidence of ectopic pregnancy has been estimated at $2 \%$ [1]. Owing to the advances in transvaginal ultrasonography, ectopic pregnancy can be diagnosed early and treated using medical rather than surgical methods. Methotrexate (MTX), an antifolate cytotoxic drug, was first used for treating a clinically stable ectopic pregnancy in 1982. Since then, it has been accepted as a reliable non-surgical method for treating ectopic pregnancies [2]. However, ectopic pregnancy can persist and progress, at a rate of 9-27\%, even after MTX treatment. Therefore, patient selection for this treatment needs to be appropriate $[3,4]$. MTX can be administered orally, systemically, or locally in the area of the ectopic pregnancy. However, systemic use is preferred over other modes of ad- ministration. Systemic MTX treatment can involve multi-dose and single-dose protocols. Due to the fact that single-dose protocols are associated with fewer adverse effects and require a smaller amount of the drug, they are currently the most popular choice for treating ectopic pregnancies. In this protocol, MTX is administered intramuscularly $\left(50 \mathrm{mg} / \mathrm{m}^{2}\right)$, and $\beta$-human chorionic gonadotropin ( $\mathrm{hCG}$ ) level is monitored on days 4 and 7 after administration. If the decrease in $\beta$-hCG concentration on days 4 and 7 exceeds $15 \%$, the level is monitored by patient follow-up on a weekly basis. Due to unknown reasons, serum $\beta$-hCG level can increase until day 4 after MTX administration $[5,6]$. The $\beta$-hCG level on day 7 is the most important criterion to determine whether the single-dose protocol was successful. Regardless, the success 
of the therapy cannot be conclusively determined, and the decision about the administration of a repeat dose cannot be made until protocol completion.

\section{OBJECTIVES}

The purpose of the study was to investigate the importance of $\beta$-hCG level evaluation on day 4 following MTX administrations, and the differences between $\beta$-hCG levels assessed on days 0 and 4 for predicting treatment success. We also aimed to analyze the success of MTX treatment in the early weeks of gestation.

\section{MATERIAL AND METHODS}

Between 2008 and 2014, 234 patients were admitted to the Department of Gynecology and Obstetrics, Ondokuz Mayıs University, due to tubal ectopic pregnancy. Of these patients, 166 received surgical and 68 received single-dose MTX treatment. The inclusion criteria for MTX treatment were as follows: (1) extrauterine gestational sac or absence of an intrauterine pregnancy despite $\beta$-hCG level of $\geq 1500 \mathrm{mIU} / \mathrm{mL}$ and a continuous increase in the levels; (2) absence of acute abdomen or hemoperitoneum; (3) absence of fetal heartbeat in the extrauterine sac; (4) $\beta$-hCG level of $<10000 \mathrm{mlU} / \mathrm{mL}$ and size of the extrauterine gestational sac of $<3.5 \mathrm{~cm}$; (5) absence of hepatic dysfunction (high levels of liver function tests) or renal insufficiency (creatinine level of $>1.5 \mathrm{mg} / \mathrm{dL}$ ); (6) absence of a known allergy to MTX; and (7) patient consent.

Patients who met these criteria received single-dose MTX treatment. One day before MTX administration (day $0)$, we performed liver and renal function tests, as well as blood count and $\beta$-hCG level measurements. The day of MTX administration $\left(50 \mathrm{mg} / \mathrm{m}^{2}\right)$ was considered day 1 . Thereafter, $\beta$-hCG levels were measured on days 4 and 7 . If the decrease in $\beta$-hCG levels was $>15 \%$ between days 4 and 7 , the patients were followed-up on a weekly basis until $\beta$-hCG levels could not be detected anymore. The second dose of MTX was administered if the patient condition was stable and a decrease in $\beta$-hCG levels was $<15 \%$. Surgery was performed if patient condition was unstable (acute pelvic pain or indicating tubal rupture), or if the patient refused the second dose of MTX. The treatment was considered successful if the condition resolved completely with a single dose of MTX and without any requirement for surgical treatment or the second dose. Serum $\beta$-hCG levels were determined using automated electrochemiluminescence immunoassays (Roche Hitachi Modular Analytics E170). Local Ethics Committee approved of the study.

\section{Statistical analysis}

Number Cruncher Statistical System (NCSS) 2007 and Power Analysis and Sample Size (PASS) 2008 (Utah, USA) were used for statistical analyses. Student's $t$ test and the definitive statistical methods (methods to calculate the mean, standard deviation, median, rate, and frequency) were used to compare normally distributed data. The U Mann-Whitney test was used to compare non-normally distributed data. Diagnostic screening tests (specificity, sensitivity, etc.) and receiver operating characteristic $(\mathrm{ROC})$ curve analysis were performed to evaluate the changes in $\beta$-hCG levels during follow-up. The results were evaluated by determining a $95 \%$ confidence interval. The $p$-value of $<0.05$ was considered as statistically significant.

\section{RESULTS}

A total of 68 patients (29.1\%) received single-dose MTX treatment. Out of them, 51 (75\%) responded to treatment. The remaining 17 patients (25\%) did not respond to treatment: multi-dose MTX treatment was administered to 7 , while 10 received surgical treatment.

Patients who did not respond to treatment formed a group in which single-dose MTX treatment was unsuccessful. Mean patient age was $31 \pm 5$ years (mean \pm SD) and $30 \pm 5$ years for those who underwent surgery and those who received a single dose of MTX, respectively. There were significant differences in serum $\beta$-hCG levels on day 0 between these two groups. Median preoperative $\beta$-hCG level in patients who underwent surgery was $2800 \mathrm{mlU} / \mathrm{mL}$ (range: $116-59769 \mathrm{mIU} / \mathrm{mL}$ ), and median $\beta$-hCG value on day 0 (i.e. before MTX treatment) in patients who received MTX was $1117 \mathrm{mIU} / \mathrm{mL}$ (range, 47-9319 mlU/mL) $(p<0.01)$.

The success rate of single-dose MTX treatment in our clinic was $75 \%$ (51/68). Comparison of patients with and without successful response to single-dose MTX treatment ( $n=51$ and $n=17$, respectively) revealed no statistically significant differences in age, gravidity, parity, and $\beta$-hCG levels on days 0 and 4 . However, there were significant differences in $\beta$-hCG levels on day 7 between the two groups $(p<0.05)$ (Table 1).

In the group which received single-dose MTX treatment, the $\beta$-hCG level decrease on days 4 and 0 was $>15 \%$ in 30 cases (44\%), and was $<15 \%$ or increased in $38(56 \%)$ cases. Among the 51 patients in whom the MTX treatment was successful, 25 (36.8\%) showed a decrease in $\beta$-hCG level of $>15 \%$ on days 0 and 4 . Sensitivity and specificity of the test were $49 \%$ and $70 \%$, respectively. The positive predictive value was $83 \%$, the negative predictive value was $31 \%$, and the accuracy was $54 \%$ (Table 2 ).

When the decrease in $\beta$-hCG level between days 4 and 7 was analyzed in all patients receiving MTX treatment, the level on day 7 was lower than on day 4, and this decrease was $>15 \%$ and $<15 \%$ in $50(73 \%)$ and 18 patients (26\%), respectively. Among the 51 patients in whom MTX treatment was successful, 44 (64\%) showed $\beta$-hCG level decrease 
Table 1. Comparison of the patients in whom single-dose methotrexate treatment was successful to those in whom the treatment was unsuccessful

\begin{tabular}{|c|c|c|c|}
\hline & $\begin{array}{l}\text { Methotrexate treatment was } \\
\text { successful }(n=51)\end{array}$ & $\begin{array}{l}\text { Methotrexate treatment was } \\
\text { unsuccessful }(n=17)\end{array}$ & $\mathbf{P}$ \\
\hline & Mean \pm SD & Mean \pm SD & \\
\hline \multirow{2}{*}{ Age } & $30.67 \pm 5.24$ & $29.94 \pm 6.89$ & 0.641 \\
\hline & Median (Min-Max) & Median (Min-Max) & $+p$ \\
\hline Gravidity & $2(1-4)$ & $1(1-4)$ & $0.042^{*}$ \\
\hline Parity & $1(0-3)$ & $0(0-3)$ & 0.052 \\
\hline$\beta$-hCG (day 0) & 1163 (range 47-9319) & 1077 (range 191-6815) & 0.772 \\
\hline$\beta$-hCG (day 4) & 806 (range 12-5470) & 1033 (range 252-5832) & 0.099 \\
\hline$\beta-h C G($ day 7$)$ & 370 (range 3-4150) & 1935 (range 191-16466) & $0.004^{* *}$ \\
\hline
\end{tabular}

Student-t Test; + U Mann-Whitney Test; ${ }^{*} \mathrm{p}<0.05 ;{ }^{* *} \mathrm{p}<0.01$

Table 2. Change in $\beta$-hCG on day 4 and day 0 after single dose methotrexate treatment

\begin{tabular}{|c|c|c|c|c|c|c|c|}
\hline & \multicolumn{6}{|c|}{ Single-dose methotrexate treatment } \\
\hline & & \multicolumn{2}{|c|}{ Successful } & \multicolumn{2}{|c|}{ Unsuccessful } & \multicolumn{2}{|c|}{ Total } \\
\hline & & $\mathbf{n}$ & $\%$ & $\mathbf{n}$ & $\%$ & $\mathbf{n}$ & $\%$ \\
\hline \multirow{3}{*}{$\begin{array}{l}\text { Change in } \beta \text {-hCG on day } \\
4 \text { and day } 0\end{array}$} & $>\% 15$ decrease & 25 & 36.8 & 5 & 7.4 & 30 & 44.1 \\
\hline & $\leq \% 15$ decrease or increase & 26 & 38.2 & 12 & 17.6 & 38 & 55.9 \\
\hline & Total & 51 & 75.0 & 17 & 25.0 & 68 & 100.0 \\
\hline \multicolumn{2}{|l|}{ Sensitivity } & & & \multicolumn{2}{|l|}{49} & & \\
\hline \multicolumn{2}{|l|}{ Specificity } & & & \multicolumn{2}{|c|}{70} & & \\
\hline \multicolumn{2}{|l|}{ Positive prediction value } & & & \multicolumn{2}{|c|}{83} & & \\
\hline \multicolumn{2}{|l|}{ Negative prediction value } & & & \multicolumn{2}{|c|}{31} & & \\
\hline \multicolumn{2}{|l|}{ Accuracy } & & & \multicolumn{2}{|c|}{54} & & \\
\hline
\end{tabular}

Table 3. Change in $\beta$-hCG on day 7 and day 4 after single dose methotrexate treatment

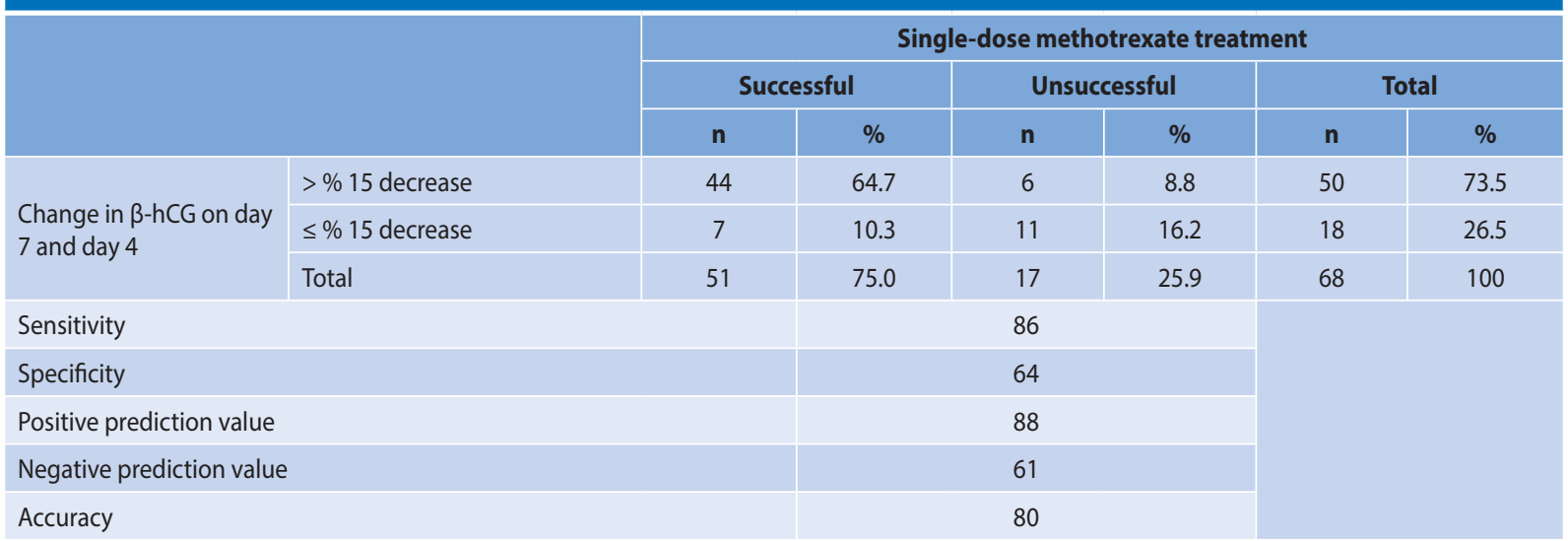

of $>15 \%$ on days 4 and 7 . The sensitivity and specificity of the test were $86 \%$ and $64 \%$, respectively. The positive predictive value was $88 \%$, the negative predictive value was $61 \%$, and the accuracy was $80 \%$ (Table 3 ).

For the subjects in whom the $\beta$-hCG decrease was $>15 \%$ on days 4 and 7 , the standard error was $6.5 \%$, and the area under the ROC curve was $81 \%$, and the corresponding values for days 0 and 4 were $7 \%$ and $64 \%$, respectively. The $15 \%$ decrease between days 7 and 4 is an efficient predictor of the success of MTX treatment (Figure 1).

\section{DISCUSSION}

Early prediction of the outcomes of single-dose MTX treatment by measuring $\beta$-hCG between days 0 and 4 may 


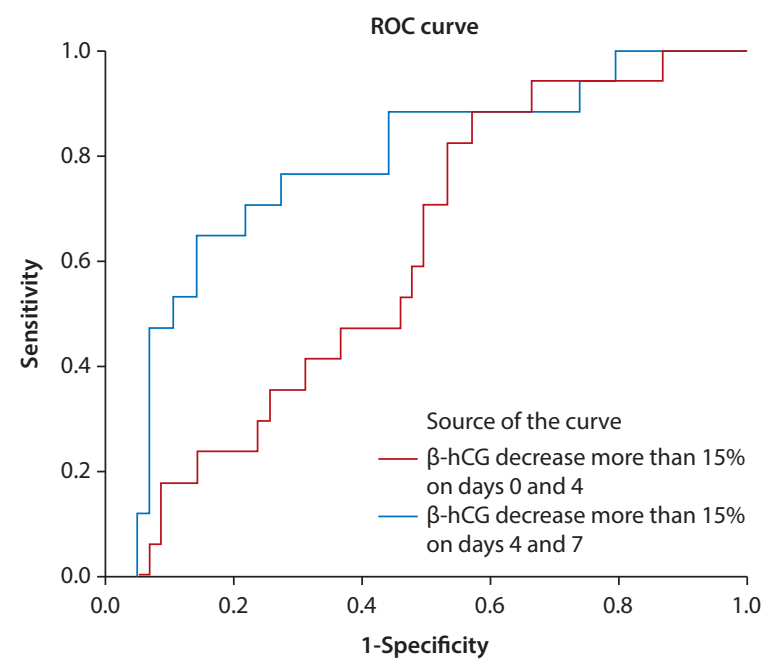

Figure 1. The area under the receiver operating characteristic curve

help to eliminate the necessity of a close 7-day follow-up of patients with predicted success. Also, it may help to determine the requirement of a second dose or more aggressive treatment methods in patients showing unfavorable outcomes without delay. In this retrospective study, we aimed to evaluate the decrease in the level of $\beta$-hCG between days 0 and 4 (early $\beta$-hCG assessment), so as to use this measurement in predicting the success of single-dose MTX treatment. We found that a decrease of $>15 \%$ in $\beta$-hCG levels between days 0 and 4 does not seem to be a better predictor for success of single-dose MTX treatment for ectopic pregnancy than between days 4 and 7. A statistically significant difference was observed only in $\beta$-hCG levels on day 7 in both, successful and unsuccessful single-dose MTX groups.

In our study, the sensitivity of the $>15 \%$ decrease in $\beta$-hCG levels between days 0 and 4 for predicting the success of single-dose MTX treatment was $49 \%$, while the positive predictive value was $83 \%$. With regard to the $\beta$-hCG level decrease of $>15 \%$ between days 7 and 4 , the sensitivity of this predictor for treatment success was $86 \%$, and the positive predictive value was $88 \%$.

In the literature, the reported success rate of single-dose MTX treatment ranges between $65-96 \%[3,7,8]$. The treatment success rate in our study (75\%) was similar to the success rates reported in 2010 by Nguyen et al. (76\%) [9], and by Skubiz et al. (77\%) [10]. However, in the study by Nguyen et al., the predictive value of the $\beta$-hCG level decrease between days 0 and 4 for treatment success was very high. In their study, the success rate of single-dose MTX treatment in all patients in whom $\beta$-hCG levels decreased between days 0 and 4 (12 patients) was $100 \%$, and they concluded that follow-up until day 7 may not be required in these patients [9]. Skubitz et al., stated that $\beta$-hCG levels decreased between days 0 and 4 in 29 out of 33 patients who were successfully treated with a single dose of MTX ( $88 \%$ success rate), and this is a clinical indicator for predicting the success of single-dose MTX treatment, with the likelihood of $88 \%$ [10].

When the decrease between the values for days 0 and 4 was taken into consideration, the probability of determining treatment success on the basis of $\beta$-hCG level decrease of $>15 \%$ was $83 \%$. This result suggests that although the decrease between days 0 and 4 is important for predicting the success of single-dose MTX treatment, it is not a better predictor than $\beta$-hCG level calculated between days 4 and 7 .

Agostini et al., reported a probability of $97 \%$ for days 0 and 4 . However, the predictor used in their study was a $\beta$-hCG level decrease of $>20 \%$ between days 0 and 4 , and the high probability of success achieved with this predictor can be attributed to the higher percentage of the decrease considered [11].

\section{CONCLUSIONS}

When $\beta$-hCG levels on days 0,4 , and 7 are considered as predictors of treatment success, significant differences are observed only between day 7 values in groups showing successful and unsuccessful outcomes. Early $\beta$-hCG assessment on days 0 and 4 appears to be a poor predictor for success of single-dose MTX treatment in ectopic pregnancy.

\section{REFERENCES}

1. Stenchever MA, DroegemuellerW, Herbst AL, [et al.]. Comprehensive Gynecology. $4^{\text {nd }}$ ed. Mosby Publishers, St. Louis 2001.

2. Tanaka T, Hayashi H, Kutsuzawa T, [et al.]. Treatment of interstitial ectopic pregnancy with methotrexate: report of a successful case. Fertil Steril.1982, 37 (6), 851-852.

3. Bixby $S$, Tello R, Kuligowska E. Presence of a yolk sac on transvaginal sonography is the most reliable predictor of single-dose methotrexate treatment failure in ectopic pregnancy. J Ultrasound Med. 2005, 24 (5), 591-598.

4. Potter MB, Lepine LA, Jamieson DJ. Predictors of success with methotrexate treatment of tubal ectopic pregnancy at Grady Memorial Hospital. Am J Obstet Gynecol. 2003, 188 (5), 1192-1194.

5. Natale A, Candiani M, Barbieri M, Calia C, Odorizzi MP, Busacca M. Preand post-treatment patterns of human chorionic gonadotropin for early detection of persistence after a single dose of methotrexate for ectopic pregnancy. Eur J Obstet Gynecol Reprod Biol. 2004, 117 (1), 87-92.

6. Natale A, Busacca M, Candiani M, [et al.]. Human chorionic gonadotropin patterns after a single dose of methotrexate for ectopic pregnancy. Eur J Obstet Gynecol Reprod Biol. 2002, 100 (2), 227-230.

7. Stovall TG, Ling FW. Single dose MTX: an expanded clinical trial. Am J Obstet Gynecol. 1993, 168, 1759-1762.

8. StovallTG, Ling FW, Gray LA. Single dose methotrexate for the treatment of ectopic pregnancy. Obstet Gynecol. 1991, 77 (5), 754-757.

9. Nguyen Q, Kapitz M, Downes K, Silva C. Are early human chorionic gonadotropin levels after methotrexate therapy a predictor of response in ectopic pregnancy? Am J Obstet Gynecol. 2010, 202 (6), 630.

10. Skubisz $M$, Lee J, Wallace $E$, Tong $S$. Decline in $\beta$ hCG levels between days 0 and 4 after a single dose of methotrexate for ectopic pregnancy predicts treatment success: a retrospective cohort study. BJOG. 2011, 118 (13), 1665-1668.

11. Agostini A, Blanc K, Ronda I, Romain F, Capelle M, Blanc B. Prognostic value of human chorionic gonadotropin changes after methotrexate injection for ectopic pregnancy. Fertil Steril. 2007, 88 (2), 504-506. 